\title{
IMPG2-Associated Retinitis Pigmentosa Displays Relatively Early Macular Involvement
}

\author{
Ramon A. C. van Huet, ${ }^{1}$ Rob W. J. Collin, ${ }^{2,3}$ Anna M. Siemiatkowska, ${ }^{2,3}$ Caroline C. W. Klaver, ${ }^{4}$ \\ Carel B. Hoyng, ${ }^{1}$ Francesca Simonelli, ${ }^{5}$ Muhammad I. Khan, ${ }^{2,6}$ Raheel Qamar, ${ }^{6,7}$ Eyal Banin, ${ }^{8}$ \\ Frans P. M. Cremers, ${ }^{2,3}$ Thomas Theelen, ${ }^{1}$ Anneke I. den Hollander, ${ }^{1}$ L. Ingeborgh van den Born, ${ }^{9}$ \\ and B. Jeroen Klevering ${ }^{1}$
}

\footnotetext{
${ }^{1}$ Department of Ophthalmology, Radboud University Medical Center, Nijmegen, The Netherlands

${ }^{2}$ Department of Human Genetics, Radboud University Medical Center, Nijmegen, The Netherlands

${ }^{3}$ Radboud Institute for Molecular Life Sciences, Radboud University Medical Center, Nijmegen, The Netherlands

${ }^{4}$ Erasmus University Rotterdam Medical Center, Rotterdam, The Netherlands

${ }^{5}$ Department of Ophthalmology, Seconda Università degli Studi di Napoli, Naples, Italy

${ }^{6}$ Department of Biosciences, COMSATS Institute of Information Technology, Islamabad, Pakistan

${ }^{7}$ Shifa College of Medicine, Islamabad, Pakistan

${ }^{8}$ Department of Ophthalmology, Hadassah-Hebrew University Medical Center, Jerusalem, Israel

${ }^{9}$ The Rotterdam Eye Hospital, Rotterdam, The Netherlands
}

Correspondence: B. Jeroen Klevering, Department of Ophthalmology, Radboud University Medical Center, Philips van Leydenlaan 15, 6525 EX Nijmegen, The Netherlands;

Jeroen.Klevering@Radboudumc.nl.

Submitted: February 10, 2014

Accepted: May 19, 2014

Citation: van Huet RAC, Collin RWJ, Siemiatkowska AM, et al. IMPG2-associated retinitis pigmentosa displays relatively early macular involvement. Invest Ophthalmol Vis Sci. 2014;55:3939-3953. DOI:10.1167/ iovs.14-14129
Purpose. To provide the first detailed clinical description in patients with RP caused by recessive mutations in IMPG2.

Methods. This international collaborative study includes 17 RP patients with inherited retinal disease caused by mutations in IMPG2. The patients were clinically (re-)examined, including extensive medical history taking, slit-lamp biomicroscopy, ophthalmoscopy, perimetry, ERG, optical coherence tomography (OCT), fundus autofluorescence (FAF) imaging, fundus photography, and color vision tests. The main outcome measures included mean age at onset, initial symptom, best-corrected visual acuity, fundus appearance, perimetry results, ERG responses, OCT images, FAF imaging, color vision test reports and DNA sequence variants.

RESULTs. The mean age at onset was 10.5 years (range, 4-20 years). Initial symptoms included night blindness in $59 \%$ of patients, a decreased visual acuity in $35 \%$, and visual field loss in $6 \%$. Fundus abnormalities were typical of RP: optic disc pallor, attenuated vessels, bone spicules, and generalized atrophy of the retina and choriocapillaris. Additionally, we observed macular abnormalities in all patients, ranging from subtle mottling of the macular pigment epithelium (two patients) and a bull's eye maculopathy (seven patients) to macular chorioretinal atrophy (seven patients).

Conclusions. Mutations in IMPG2 cause a severe form of RP with symptoms manifesting in the first 2 decades of life. IMPG2-associated $\mathrm{RP}$ is frequently accompanied by macular involvement, ranging from mild pigment alterations to profound chorioretinal atrophy. The resulting decrease in central vision in combination with the severe tunnel vision leads to severe visual impairment in patients with IMPG2-associated RP.

Keywords: Retinitis pigmentosa, IMPG2, macular atrophy, bull's eye maculopathy, natural course.

\section{$\mathbf{R}$} etinitis pigmentosa (RP) is a group of diseases featuring progressive degeneration of rod and cone photoreceptor cells and RPE, and is considered the most commonly inherited retinal dystrophy with an estimated prevalence of approximately $1: 4000 .^{1-3}$ Retinitis pigmentosa typically starts with night blindness followed by loss of the peripheral visual field that leads to tunnel vision, whereas visual acuity often remains normal until the late stages. ${ }^{3,4}$ Hallmark fundus abnormalities in RP are bone-spicule pigmentation, a waxy pale optic disc, and attenuation of retinal vessels. Electroretinography (ERG) responses reveal rod and cone dysfunction, where rod abnormalities often are observed earlier in the course of the disease. However, wide variability in terms of disease onset, progression rate, and degeneration patterns are observed in $\mathrm{RP}^{4,5}$

The genetic background underlying RP is also very heterogeneous. Inheritance modes observed in RP include autosomal-recessive (30\% of patients), autosomal-dominant (20\%), X-linked (10\%), mitochondrial $(<1 \%)$ patterns, and a few cases of digenic RP. ${ }^{6-9}$ However, the remaining $40 \%$ of patients are isolated cases. ${ }^{10}$ Autosomal recessive RP is currently associated with mutations in 42 different genes (RetNet, available in the public domain at https://sph.uth.edu/ retnet/), which provide a molecular genetic explanation for approximately $50 \%$ of all recessive RP cases. ${ }^{11}$ The proteins encoded by these genes are involved in a broad range of cellular 
functions, including phototransduction, the visual (retinoid) cycle, transport along the connecting cilium, cell-to-cell signaling or synaptic interaction, gene regulation, cell or cytoskeletal structure, cell-cell interactions, and outer segment phagocytosis..$^{4,10,12}$

Recently, mutations in the IMPG2 gene have been implicated in autosomal recessive RP. ${ }^{13}$ This gene encodes the interphotoreceptor matrix proteoglycan-2 (IMPG2), formerly known as IPM 200 or SPACRCAN, ${ }^{14}$ which is localized in the retinal extracellular matrix (also known as the interphotoreceptor matrix [IPM]). The IPM is a viscous substance mainly composed of glycoproteins and proteoglycans that fills the space between individual photoreceptor cells and between photoreceptors and the RPE. ${ }^{15,16}$ For many years, the IPM was considered merely a fixating medium, ${ }^{17}$ but in the past few decades multiple functions of the IPM have been reported, including important functions in intercellular communication, regulation of neovascularization, cell survival, membrane turnover, photoreceptor differentiation and maintenance, retinoid transport, matrix turnover, and the precise alignment of the photoreceptor cells to the optical light path. ${ }^{14,18,19}$ Both rod and cone photoreceptor cells synthesize IMPG2 and secrete the protein into the IPM, ${ }^{20}$ where it binds to other proteins, such as hyaluronan, and also seems to be anchored in the plasma membrane of the photoreceptor cells, thereby fixating the photoreceptors in the IPM. ${ }^{21}$ Additionally, IMPG2 is thought to have calcium-binding potential, which suggests it has an important role in sequestering extracellular calcium released by photoreceptors in response to light. ${ }^{21}$

Knowledge of the natural course of IMPG2-related RP is of significant importance for prognosis counseling as well as genetic counseling. Furthermore, this knowledge is vital in the view of emerging therapy trials, in terms of patient selection and the assessment of treatment effects. In this international collaborative study, we aim to provide a detailed overview of the clinical findings in patients with IMPG2-associated RP.

\section{Methods and Patients}

\section{Subjects and Genetic Analysis}

The specialized ophthalmogenetic centers of the Radboud University Medical Center ( $\mathrm{RACvH}, \mathrm{CBH}$, and $\mathrm{BJK}$ ), the Rotterdam Eye Hospital (LIvdB), the Erasmus University Medical Center Rotterdam (CCWK), the Hadassah-Hebrew University Medical Center in Jerusalem (EB), the Seconda Università degli Studi di Napoli (FS), and the Shifa College of Medicine in Islamabad (RQ) participated in this study. As described previously, ${ }^{13}$ six families of Israeli, Palestinian, Pakistani, Italian, or Dutch origin were found to carry causative mutations in IMPG2 (families A-F, Fig. 1). Additionally, we selected four more families after identification of causative IMPG2 mutations in a targeted next-generation sequencing experiment in 100 Dutch RP probands (family $\mathrm{G}),{ }^{22}$ or whole exome sequencing (families H, J, K, Fig. 1). Exome sequencing was performed using the 5500xl Genetic Analyzer of Life Technologies (Applied Biosystems, Foster City, CA, USA) and the Agilent SureSelectXT Human All Exon 50-Mb amplification kit (Agilent Technologies, Inc., Santa Clara, CA, USA). Data were analyzed with LifeScope software (version 2.1; Life Technologies, Applied Biosystems). All mutations were confirmed with Sanger sequencing.

We adhered to the tenets of the Declaration of Helsinki and obtained approval for this study from the Institutional Ethics Committee from the Radboud University Medical Center. Approval included permission to use the documented medical data and, when indicated, clinically reassess affected individuals and to obtain blood for the purposes of DNA extraction and genetic analysis. We obtained informed consent from all participants before the collection of blood samples and additional ophthalmologic examinations.

\section{Clinical Analysis}

We collected the available clinical data from the medical files of all patients. Nine patients were clinically reevaluated after the identification of causative IMPG2 mutations. Medical history was registered with a focus on the age at onset, initial symptoms, and the overall course of the retinal disorder. The age at onset was defined as the age at which the initial symptom was noticed by the patient. Additionally, we questioned patients about the presence of syndromic features, which generally occur in $20 \%$ to $30 \%$ of RP patients. ${ }^{4}$ This questionnaire included the presence of hearing and balance abnormalities, renal failure, cardiac and respiratory anomalies, polydactyly, obesity, cognitive impairment, fertility disorders, hypogonadism, and dental anomalies.

Clinical examination included best-corrected visual acuity (BCVA), slit-lamp biomicroscopy, and ophthalmoscopy. Additional examinations were performed if feasible. Goldmann (kinetic) perimetry was performed in 11 patients using targets V-4e, III-4e, I-4e, I-3e, I-2e, and I-1e. In two patients (F-II: 1 and F-II:2) perimetry was restricted to analysis of the central $30^{\circ}$ of the visual field with the Humphrey perimeter (Carl Zeiss Meditec, Jena, Germany). In all but one patient (F-II:2), fullfield ERG was performed according the guidelines of the International Society for Clinical Electrophysiology of Vision. ${ }^{23}$ Results were compared to the local reference values. We evaluated color vision in six patients using the Farnsworth Dichotomous Test (Panel D-15) and/or the Hardy-RandRittlers test. Fundus photographs of the central retina (Topcon TRC50IX; Topcon Corporation, Tokyo, Japan) were obtained in 15 patients. Fundus autofluorescence images (Spectralis; Heidelberg Engineering, Heidelberg, Germany) of the central retina were acquired in eight patients using a confocal scanning laser ophthalmoscope with an optically pumped solid state laser $(488 \mathrm{~nm})$ for excitation. Spectraldomain optical coherence tomography (SD-OCT, Spectralis; Heidelberg Engineering) could be performed in 13 patients. In three patients, a high-resolution OCT was not available and a time-domain OCT (Stratus; Carl Zeiss Meditec) was obtained. No OCT images were available for the remaining four patients. In eight Dutch patients with high-resolution SDOCT images (mean age: 51 years; range, 23-67 years), we quantified thickness of the total retina at the foveola and at $0.25,0.5,1.0,1.5,2.0$, and $2.5 \mathrm{~mm}$ eccentricity from the foveola in the right eye using the thickness graphs in the Heidelberg Eye Explorer Software (version 1.6.4.0; Heidelberg Engineering). In seven of these patients, we quantified the foveal volume by measuring the retinal volume within the central $3 \mathrm{~mm}^{2}$ using the thickness map in the Heidelberg Eye Explorer Software (version 1.6.4.0; Heidelberg Engineering). A normal dataset of retinal thickness and foveal volume in individuals without (vitreo)retinal disease was obtained from 25 age-matched Dutch individuals (mean age: 46 years; range, 27-62 years) for reference purposes.

\section{Results}

Ten families with a total of 17 affected individuals were included in this study. The pedigrees of all families are depicted in Figure 1. An overview of the clinical findings in all 17 patients is provided in Table 1. 


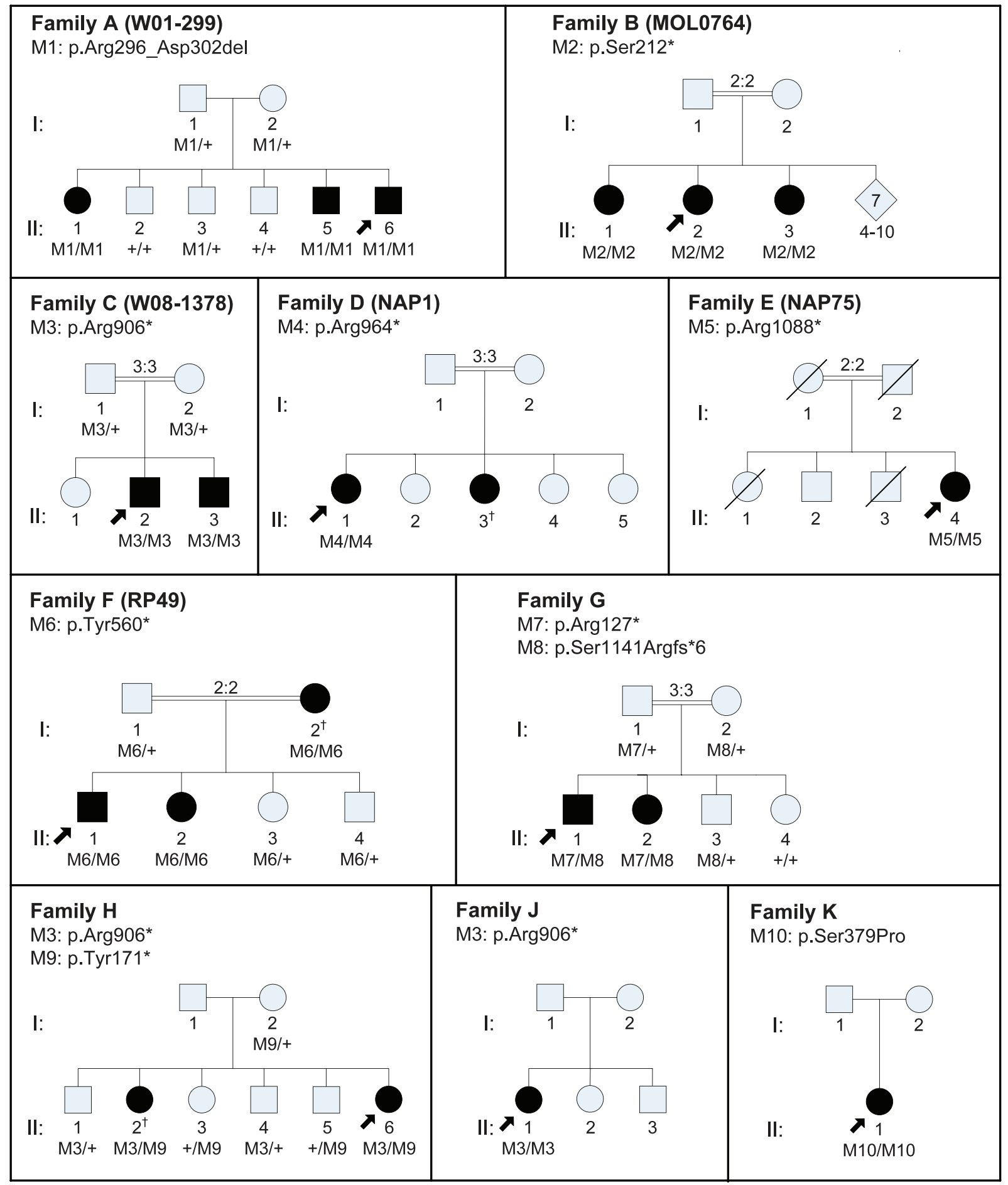

Figure 1. Pedigrees of the families that were included in this study. Where relatives were available (families A, B, C, F, G, and H), the mutation segregates with the disease. Plus signs denote the wild-type allele, square boxes indicate men, circles indicate women, and affected individuals are pointed out in black. The arrows indicate the probands. Double lines point out consanguineous marriages, the numbers indicate the degree of consanguinity. The dagger $(\dagger)$ indicates the patients diagnosed with RP not included in this study due the lack of clinical data.

\section{Clinical Evaluation}

The most recent examination of the $17 \mathrm{RP}$ patients was performed at a mean age of 49 years (range, 23-67 years). The mean age at onset was 10.5 years (range, 4-20 years), and night blindness was the most frequent initial symptom, occurring in 10 patients (59\%). Other initial symptoms were decrease in visual acuity (35\%) and loss of visual field (6\%). In the patients who initially revealed a decreased visual acuity, normal BCVA was measured before the decrease in visual acuity, which excludes refractive amblyopia. In one patient (A-II:6), the diagnosis of RP was made during a routine ophthalmologic consultation when he was 12 years old. At that time, he had not noticed any symptoms associated with RP, but later in the course of the dystrophy, night blindness manifested as the first 


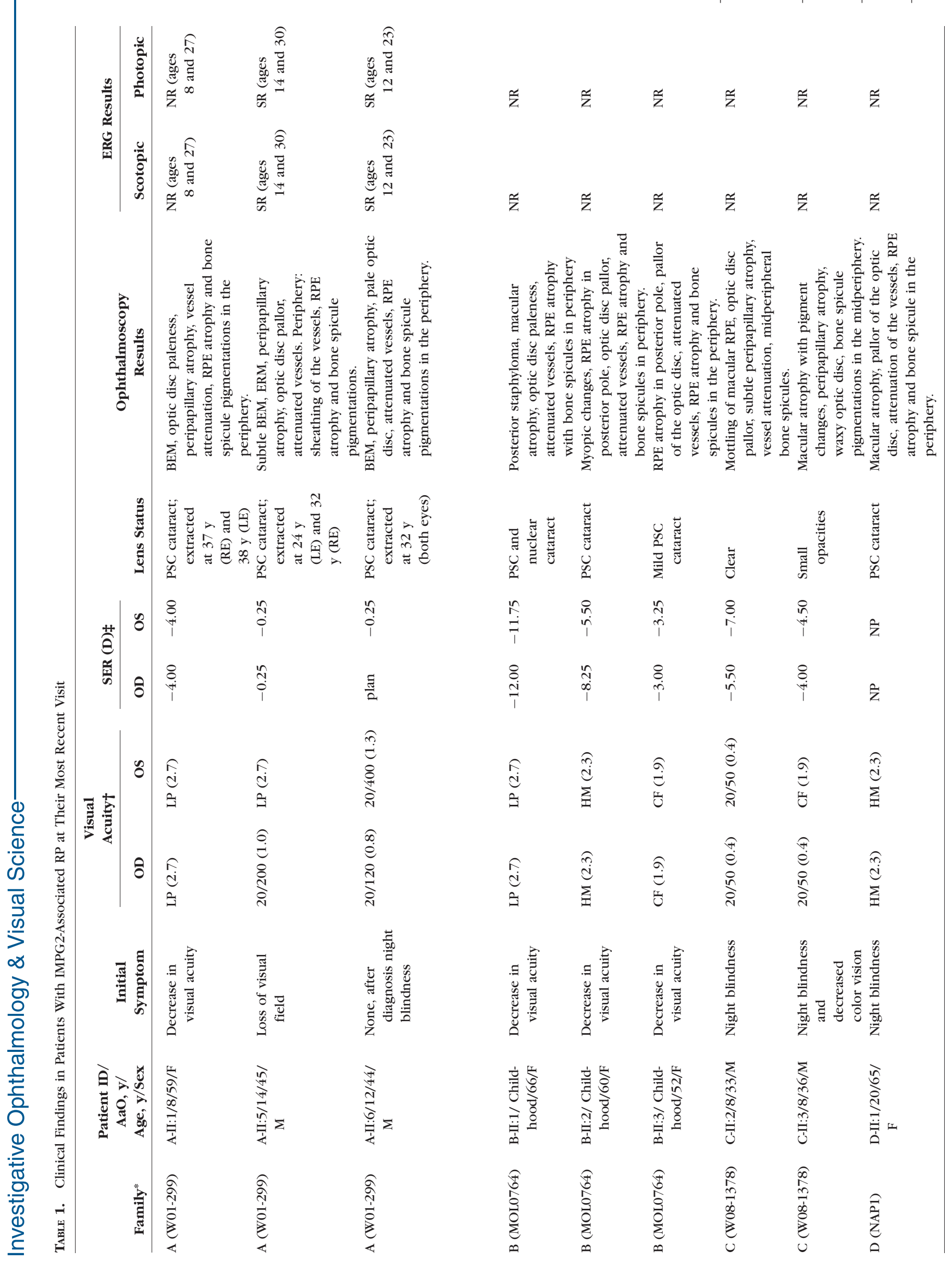




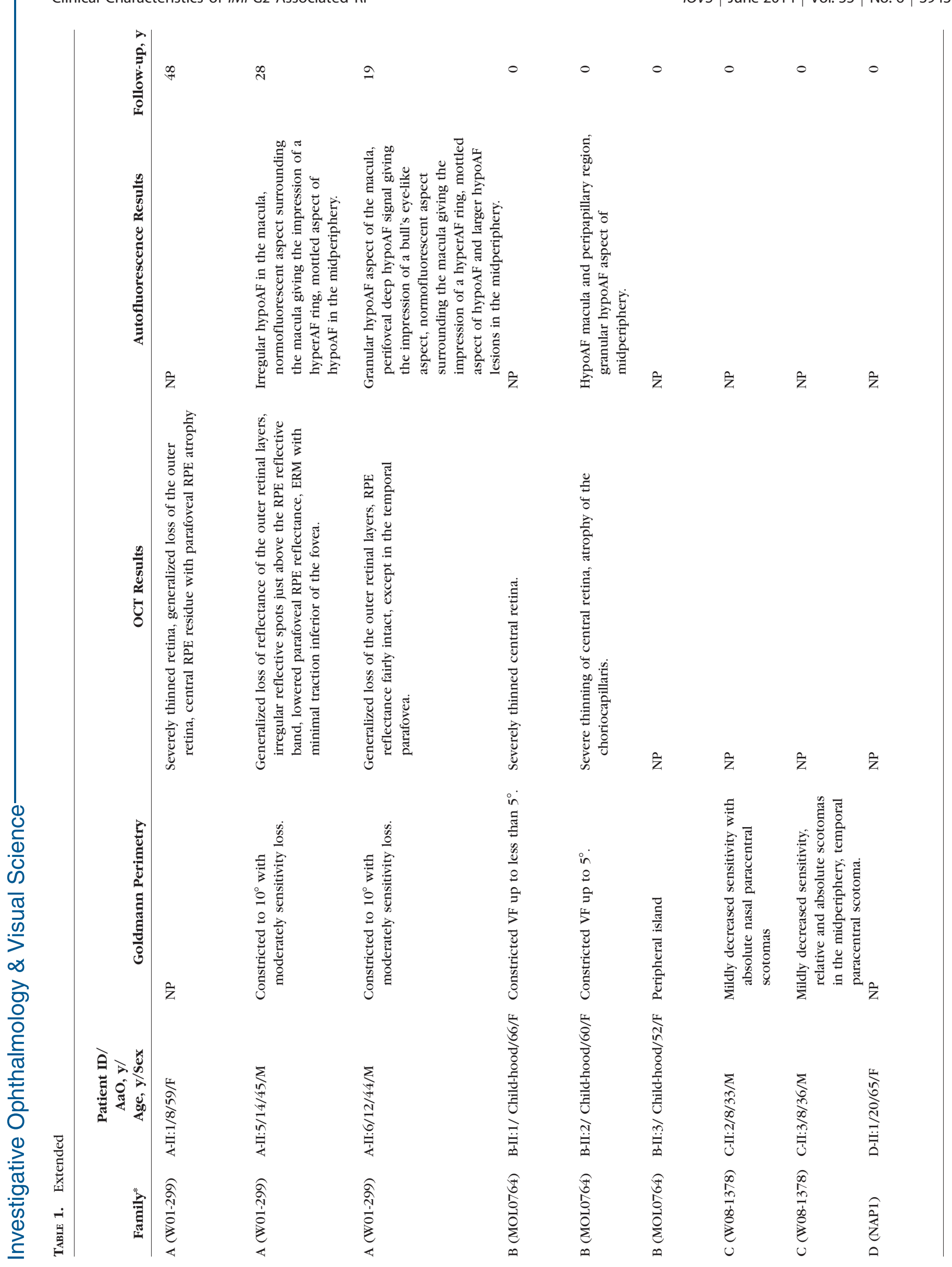




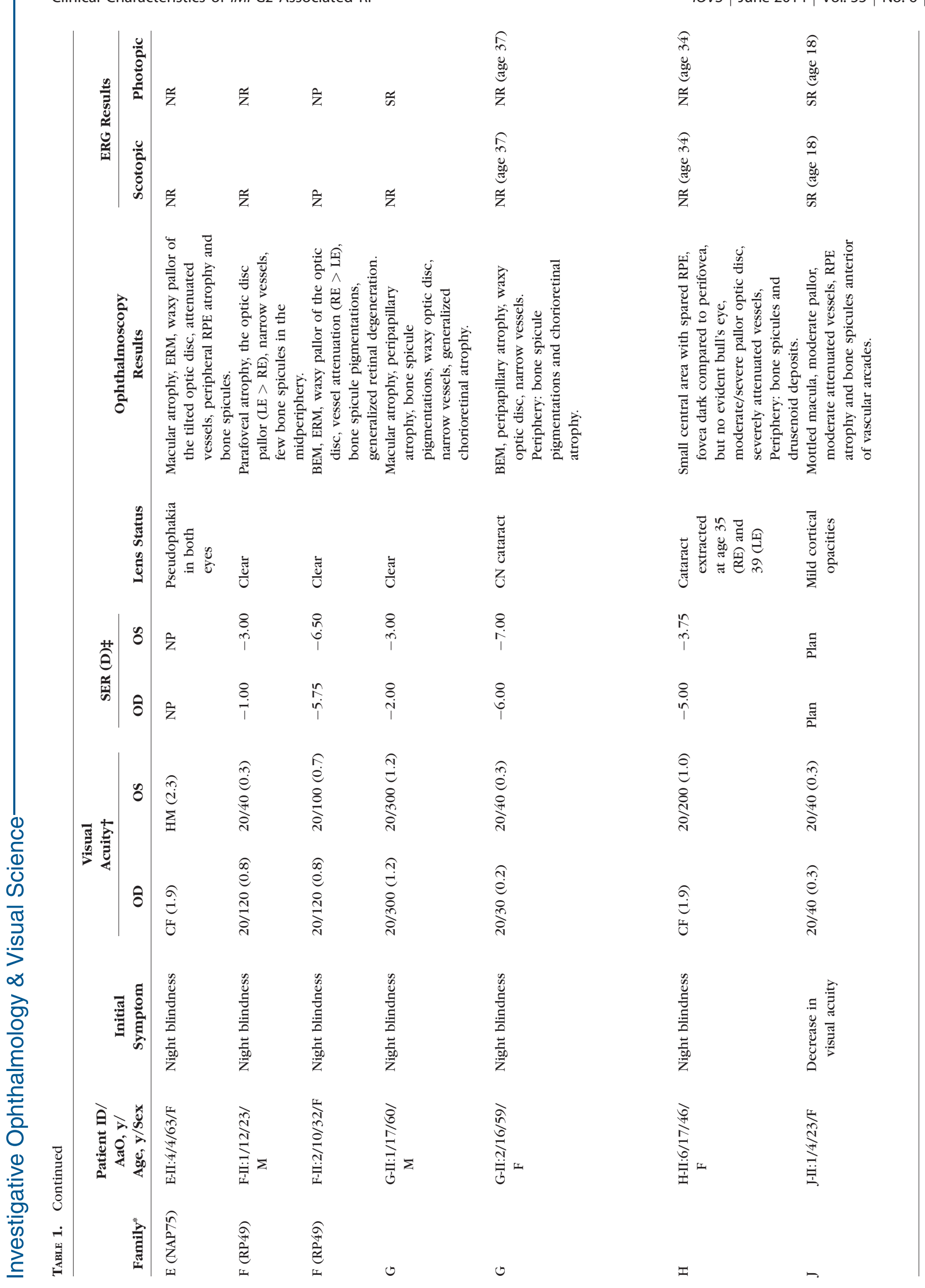




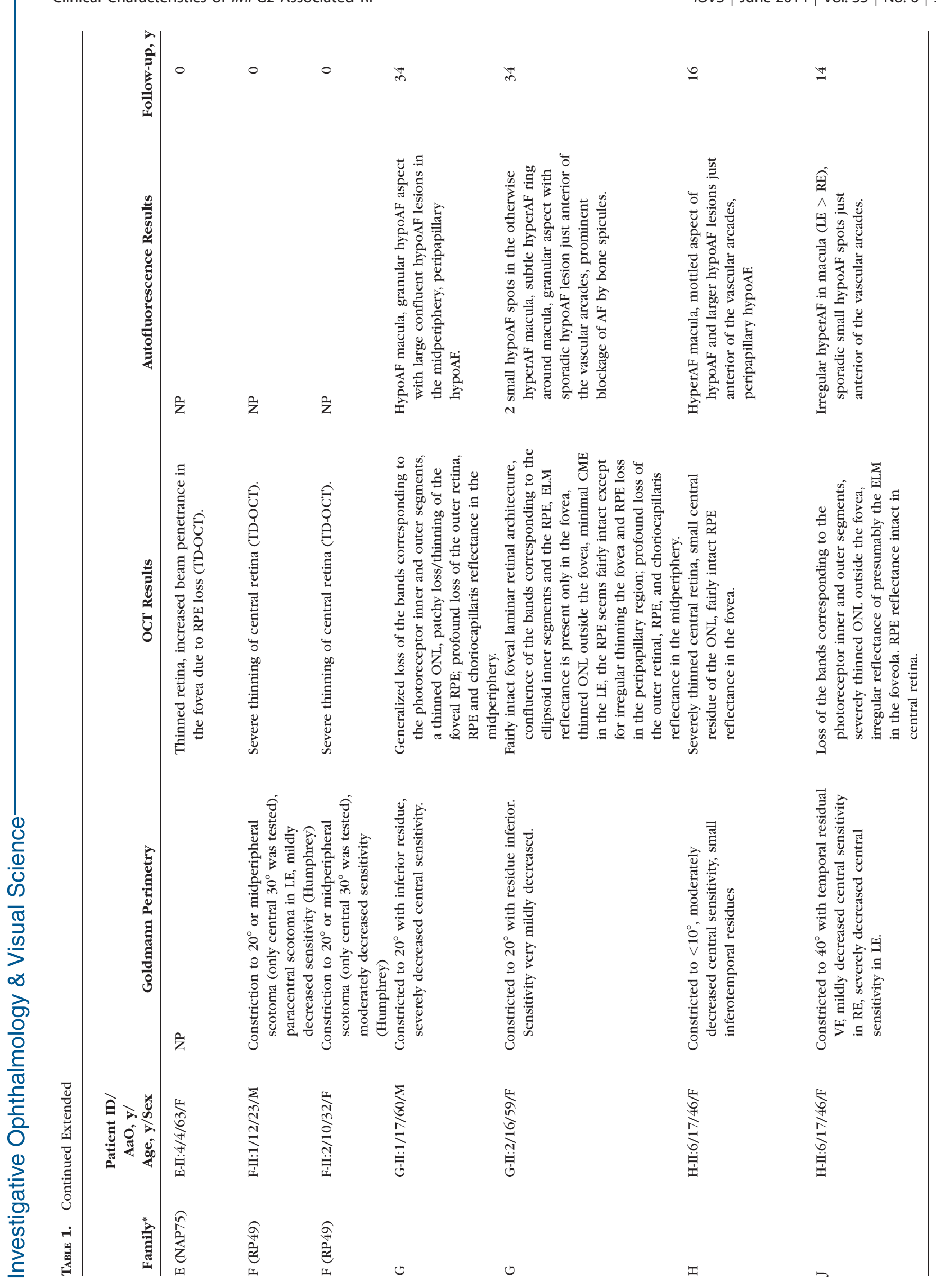



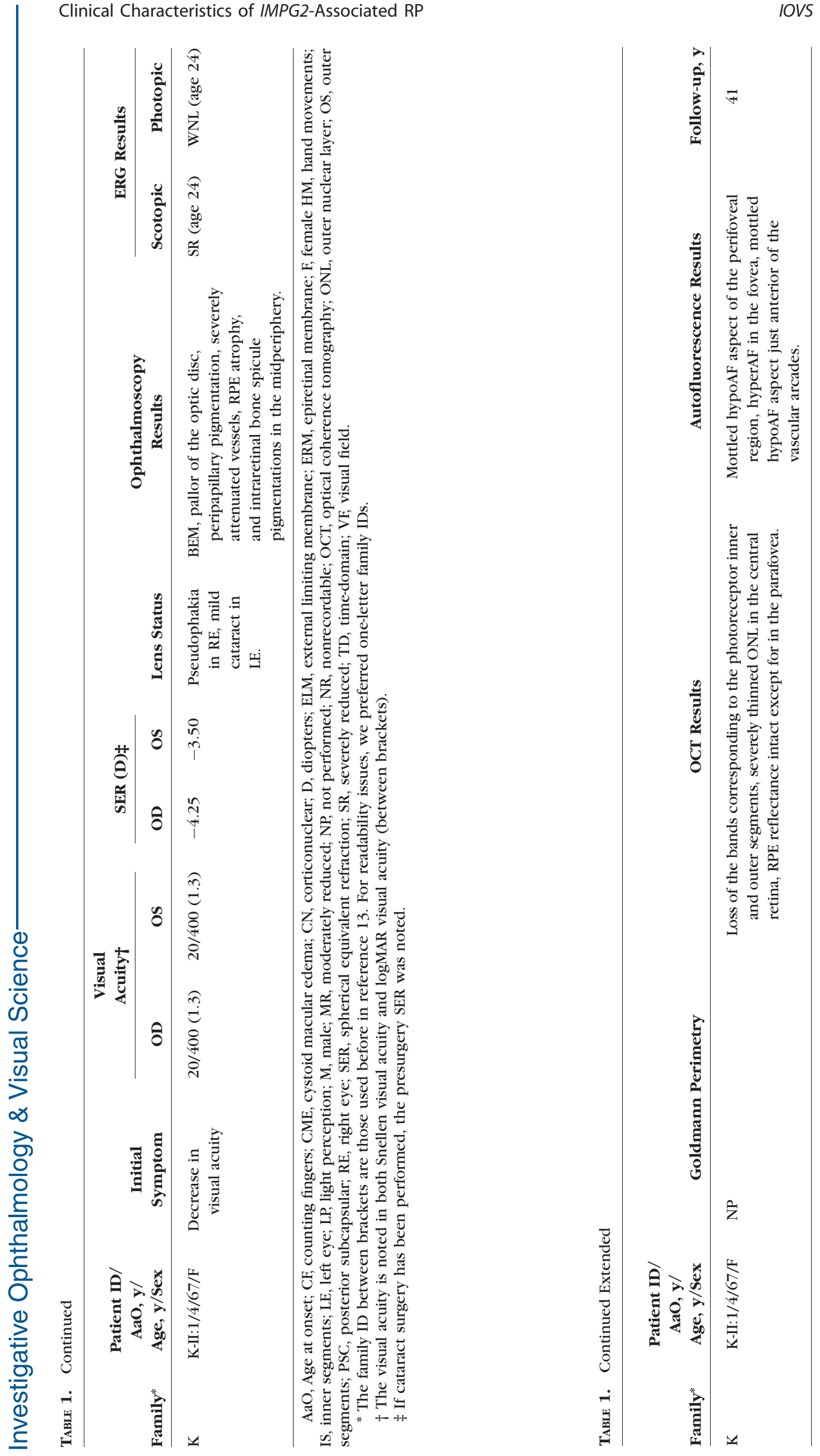


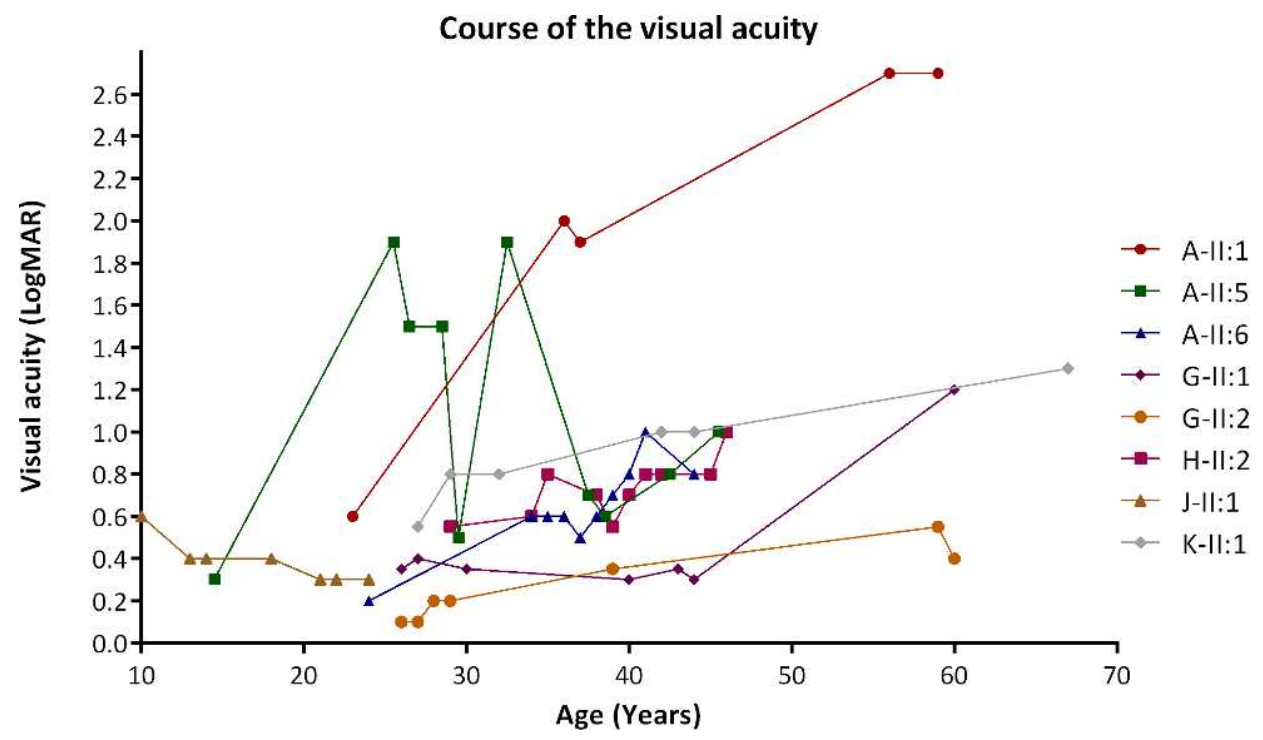

Figure 2. Graph showing the change in visual acuity ( $y$-axis) related to the age in years ( $x$-axis) in patients carrying mutations in the IMPG2 gene. Snellen visual acuity was transformed into logMAR for visualization purposes. A logMAR value of 1.9 was assigned to counting fingers (CF), 2.3 to hand movements (HM), and 2.7 to light perception (LP). When the visual acuity differed in both eyes, the visual acuity of the best eye was used. The improvement in visual acuity in patient A-II: 5 was seen after cataract surgery; subsequently, the decrease in visual acuity was probably due to cystoid macular edema, which was successfully treated. The cause of the improvement in patient J-II:1 is unclear, because refractive, optical, or retinal causes seemed absent.

symptom at age 24. In eight patients, an extended clinical follow-up period varying from 14 to 48 years was available (mean: 29 years). The course of the BCVA for each of these patients during follow-up is represented in Figure 2. None of the patients showed extraocular abnormalities that are indicative of syndromic RP.

Refractive errors included mild to high myopia (range of spherical equivalents: plan to $\mathbf{- 1 2 . 0 0}$ diopters; Table 1). Significant lens opacities were observed in 13 patients, most often subcapsular posterior cataracts (seven patients [41\%], Table 1). Six patients had experienced cataract surgery, most often in the fourth decade (five of six patients). Ophthalmoscopy revealed the classic RP features, including bone spicule pigmentation at the (mid)periphery, attenuated vessels, waxy pallor of the optic disc, and atrophy of the RPE and choriocapillaris in all RP patients. In one patient (A-II:5), marked sheathing of the peripheral retinal vessels was noted (Fig. 3A). In addition, all patients revealed macular abnormal-
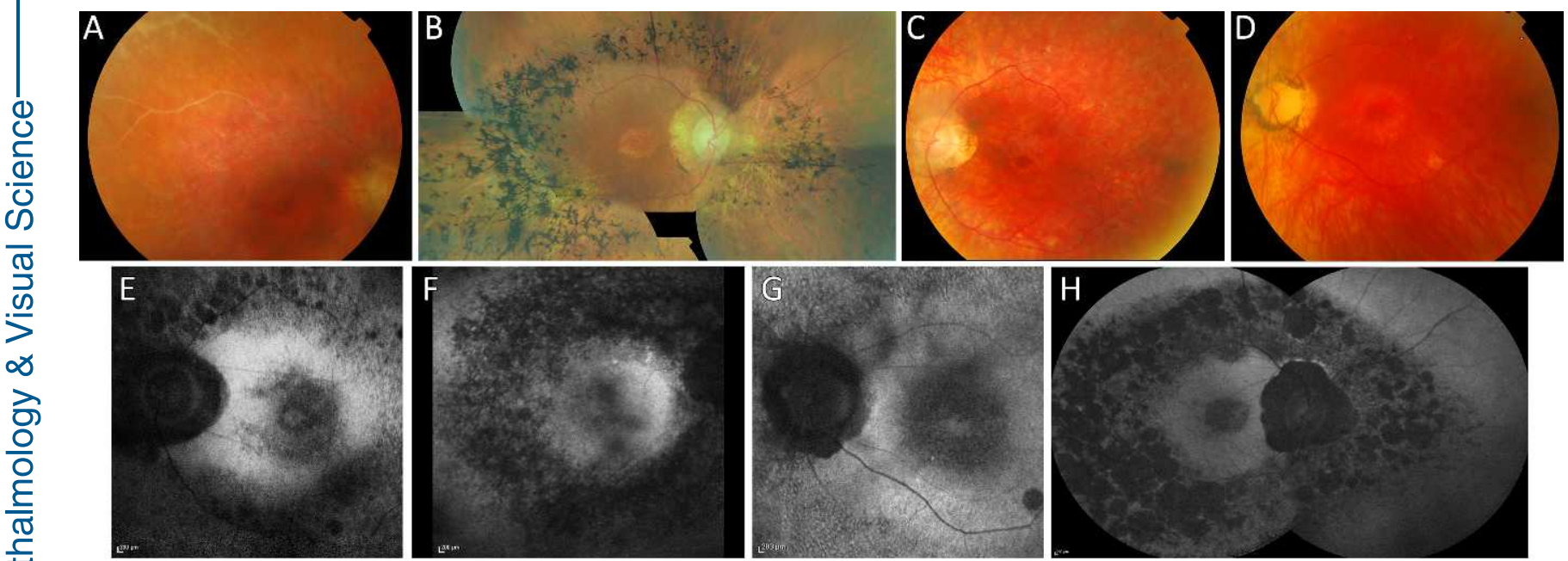

Figure 3. Fundus photographs and FAF imaging of patients carrying mutations in IMPG2. (A and F) Fundus photograph of temporal superior region of the retina (A), showing sheathing of the retinal vessels, and FAF imaging of the central retina (G), revealing irregular FAF signal in the macula and a granular hypoautofluorescent aspect of the RPE surrounding the posterior pole, in patient A-II:5 (age 45). (B and H) Fundus photograph composition (G), showing macular atrophy, and FAF images (I), revealing a hypoautofluorescent macula, a granular aspect with large confluent hypoautofluorescent lesions in the midperiphery, and peripapillary hypoautofluorescence, of patient G-II:1 (age 60). (C and E) Fundus photograph (C) and FAF image (F) of the central retina in patient A-II:6 (age 44). The fundus photograph reveals a BEM. The FAF image shows granular aspect of the macula, whereas deeper perifoveal hypoautofluorescence gives the impression of a BEM, mottled aspect of midperiphery with larger hypoautofluorescent lesions. (D and G) Fundus photograph (D) and FAF image (H) of the central retina in patient K-II:1 (age 67). The fundus photograph shows a BEM, whereas FAF reveals a mottled hypoautofluorescent aspect of the perifoveal region, hyperautofluorescence in the fovea, and a mottled aspect just anterior of the vascular arcades. 


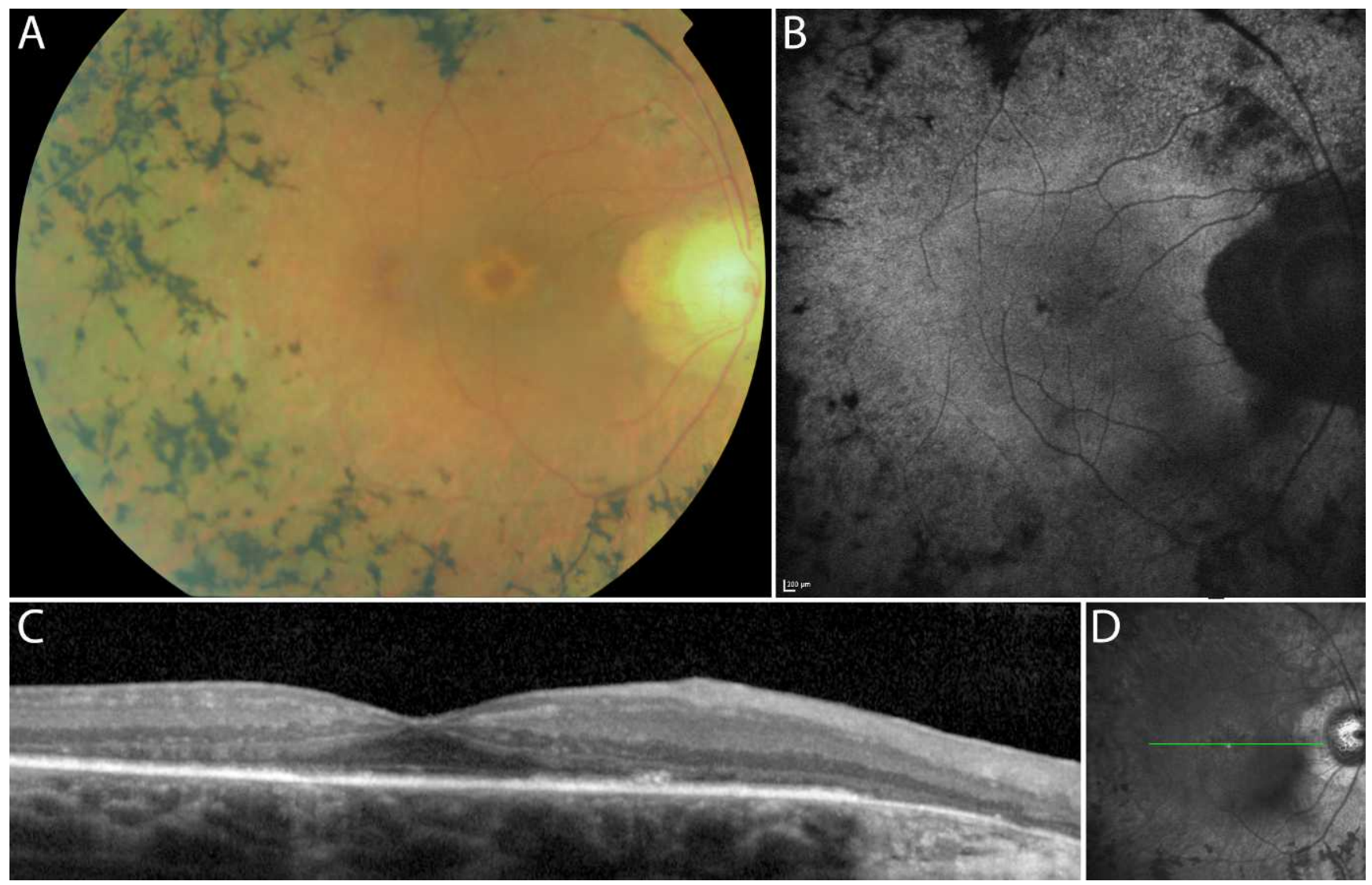

Figure 4. Multimodal imaging of the central retina in patient G-II:2 at the age of 59. (A) Fundus photograph showing a BEM, attenuated vessels, peripapillary atrophy, pale optic disc, bone-spicule pigmentations, and chorioretinal atrophy in the midperiphery. (B) Autofluorescence imaging shows a subtle hyperautofluorescent ring around the macula, spots of decreased macular and peripheral autofluorescence, and absence of autofluorescence corresponding with the peripapillary atrophy. (C) Spectral domain OCT reveals loss of the bands corresponding to the photoreceptor inner and outer segments in the macula. The external limiting membrane is present only in the fovea. The RPE layer seems fairly intact, except for irregular thinning in the fovea and loss of RPE in the peripapillary region. (D) Infrared en face image reveals the location of the SDOCT image (green line).

ities ranging from subtle changes, such as mottling of the macular RPE as observed in C-II:2 and J-II:1, to profound macular atrophy as was observed in seven patients (mean age: 57 years; range, 36-66, Fig. 3B). A bull's eye maculopathy (BEM) was a distinctive ophthalmoscopic feature in six patients, which mainly was observed during the fifth and sixth decades of life (mean age: 51 years; range, 32-67 years, Figs. $3 \mathrm{C}, 3 \mathrm{D}, 4 \mathrm{~A})$. Since the exact onset of the BEM could not be ascertained, we were unable to define the interval in which the BEM had been present in these patients. Progression of a BEM to atrophy covering the whole fovea was observed in the follow-up data of patient G-II:1.

Perimetry revealed visual field constriction resulting in tunnel vision of $20^{\circ}$ or less in nine patients (mean age: 48 years; range, 23-66 years; Fig. 5). Macular involvement was apparent by a gradual decrease in central sensitivity, which we observed in 10 patients (mean age: 40 years; range, 23-66 years). We observed paracentral scotomas in two patients (C-II:2 and CII:3) with a relatively intact (mid)peripheral visual field (Fig. 5C). Additionally, paracentral scotomas were present in patients F-II:1 and F-II:2, in whom only the central $30^{\circ}$ was analyzed with the more sensitive static perimeter (Table 1 ). Electroretinographic responses were nonrecordable in 11 patients (mean age: 51 years). In five patients (mean age: 31 years), a severely reduced photoreceptor dysfunction was seen in a rod-cone pattern. Evaluation of color vision resulted in an isolated tritan defect in patients A-II:6, G-II:1, and G-II:2 at ages
24, 40, and 39, respectively, whereas patients C-II:2 and C-II:3 demonstrated strong protan, deutan, and tritan defects at ages 33 and 36, respectively. In patient A-II:5, we did not detect color vision defects.

Fundus autofluorescence (FAF) images revealed macular involvement in all eight patients for whom FAF imaging was available. The macular aspects varied from hyperautofluorescence to profound hypoautofluorescent RPE lesions (mean age: 51 years; range, 23-67 years, Figs. 3E-G, 3I, 4B). Midperipheral changes include granular or mottled hypoautofluorescent changes that are spread to the vascular arcades. In some patients, large (confluent) hypoautofluorescent lesions were observed just anterior of the vascular arcades (Figs. 3E, $3 \mathrm{H})$. Evaluation of the central retinal structure with SD-OCT revealed loss of photoreceptors before RPE cell loss, which eventually result in moderate to severe retinal thinning (Fig. 6A). The foveal volume in seven Dutch RP patients (mean age: 49 years; range, 23-67) was significantly lower compared with the foveal volume in 25 age-matched Dutch healthy controls ( $P$ $<0.0001$; Fig. 6B). In all patients with high-resolution SD-OCT, except in G-II:2, the bands corresponding to the photoreceptor inner and outer segments were lost. ${ }^{24}$ The outer nuclear layer, containing the photoreceptor cell bodies, was concentrically lost and severely thinned where present. We observed concentric atrophy of the layer that corresponds to the RPE cells that progressed from the midperiphery. The central abnormalities in the RPE layer started in the parafoveal region, 

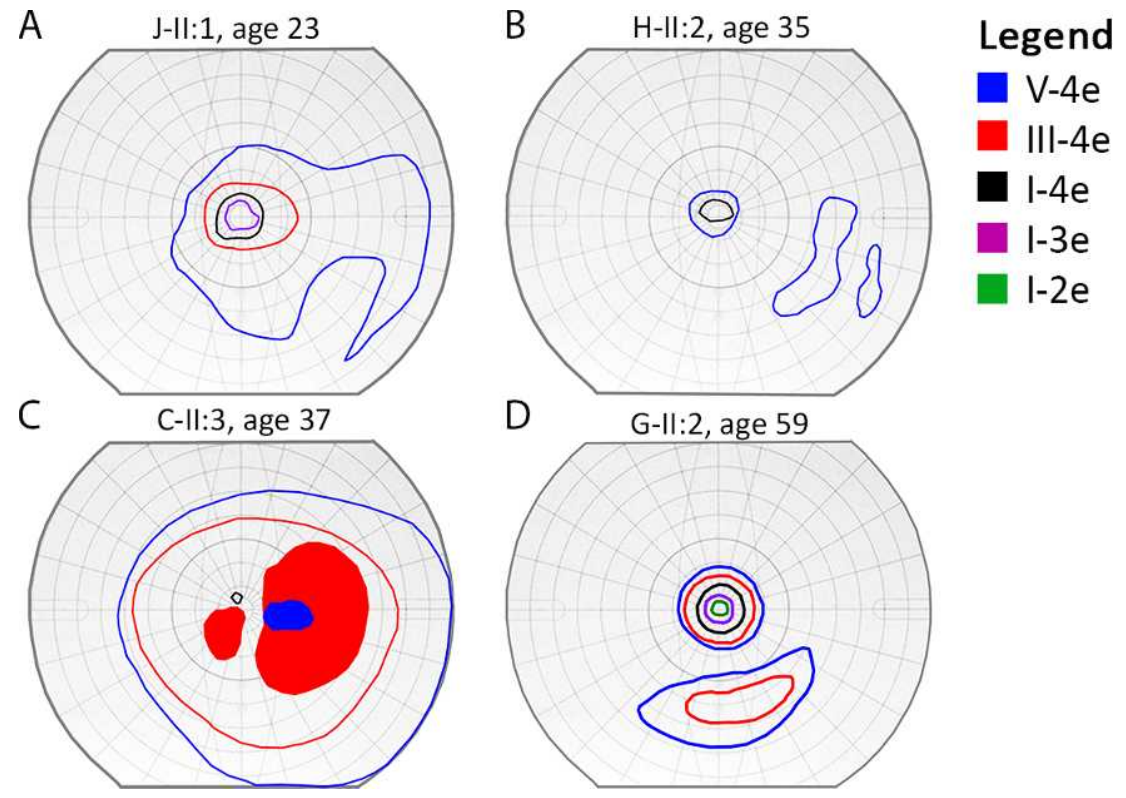

Figure 5. Goldmann perimetry findings in the right eyes of patients J-II:1 (age 23, A), H-II:2 (age 35, B) C-II:3 (age 37, C), and G-II:2 (age 59, D). The colored lines indicate the outer borders of the isopters. The filled areas indicate scotomas for the isopter of that color.

as was observed in four patients who also revealed a BEM (Fig. 6D). In patient J-II:1, who displayed mottling in the macula, the RPE appeared normal on SD-OCT (Fig. 6E). In later stages of the disease, we observed loss of the foveal RPE layer, which was highlighted by a increased beam penetration and choroidal reflection in patients E-II:4 and G-II:1 (Fig. 6F). Spectral-domain OCT scans in the midperiphery revealed loss of the photoreceptor-RPE complex and intraretinal pigment deposits in patients G-II: 1 and G-II:2 at ages 60 and 59, respectively (Fig. $6 \mathrm{G}$ ), whereas patient J-II:1 at the age of 23 revealed only photoreceptor loss (Fig. 6H).

\section{Mutation Analysis}

A description of the molecular genetic findings in families A to
F were reported earlier. ${ }^{13}$ In summary, sequence analysis of all 19 coding exons of IMPG2 led to the identification of 10 different pathogenic variants in these 17 patients (Table 2). In the families with available family members, the homozygous or compound heterozygous mutations segregated completely with the RP phenotype (Fig. 1). ${ }^{13}$

In addition to the mutations described earlier, ${ }^{13}$ four new pathogenic variants in IMPG 2 were identified. In family $\mathrm{G}$, a targeted next-generation sequencing approach that covered 111 blindness genes resulted in two compound heterozygous mutations: a nonsense mutation (p.Arg127*), which is predicted to cause premature truncation of the IMPG 2 protein, and a 4-base-pair deletion (c.3423-8_c.3423-5del) that affects the splice acceptor site (Table 2). ${ }^{22}$ Reverse transcriptase PCR analysis on RNA isolated from patients' lymphoblastoid cells revealed that, instead of the regular splice acceptor site, a second splice site located upstream in the same intron is used that results in the inclusion of 80 additional nucleotides to IMPG2 mRNA, subsequently leading to a frameshift and premature termination of IMPG2 (Supplementary Fig. S1). The cDNA products generated from RNAs isolated from cells grown under nonsense-mediated decay (NMD)-suppressing conditions show subtle differences compared with those generated from RNAs isolated from cells grown under normal conditions. Growing cells under NMD-suppressing conditions did not yield an obvious difference in the amount of aberrantly spliced IMPG2, indicating that a truncated protein may be produced. The other novel mutations include a nonsense mutation (p.Tyr171*) and a missense mutation (p.Ser379Pro). The nonsense mutation is predicted to cause a premature truncation of IMPG2. The p.Ser379Pro missense mutation changes a highly conserved amino acid and is unanimously predicted to be pathogenic by multiple in silico prediction tools (SIFT: deleterious [score: 0], Polyphen-2: probably damaging [score: 1.000], Align GVGD: Class C65, MutationTaster: disease causing [probability: 0.994], Grantham score: 74, PROVEAN prediction: deleterious [score: -2.972$]$ ).

\section{Discussion}

\section{The Phenotype of IMPG2-Associated RP}

In this study, we provide a detailed clinical description of the RP associated with mutations in IMPG2, a gene that recently was added to the long list of genes that may cause autosomal recessive RP when mutated. ${ }^{13}$ Most of the patients with IMPG2-associated RP demonstrated the classic RP symptoms: night blindness and progressive concentric loss of the visual field. However, 6 of 17 patients mentioned a decrease in BCVA that could not be attributed to amblyopia as the initial symptom. Loss of vision as the initial symptom is not just a result of our electrically illuminated nighttime environment that compensates for an impaired night vision, ${ }^{4,5}$ but a consequence of macular abnormalities that are a prominent feature of this type of RP. Overall, the BCVA progressively decreased during the first 4 decades of life, and subsequently deteriorates to levels lower than 20/300 during the fifth and sixth decades of life. The only exception was patient G-II:2, who still enjoyed a BCVA of 20/30 at the age of 59 .

Thirteen of the $17 \mathrm{RP}$ patients included in this study showed significant macular abnormalities: a BEM was observed in six patients (mean age: 51 years) and profound macular atrophy in seven patients (mean age: 57 years). We hypothesize that the perifoveal atrophy, manifesting as a bull's eye pattern, 


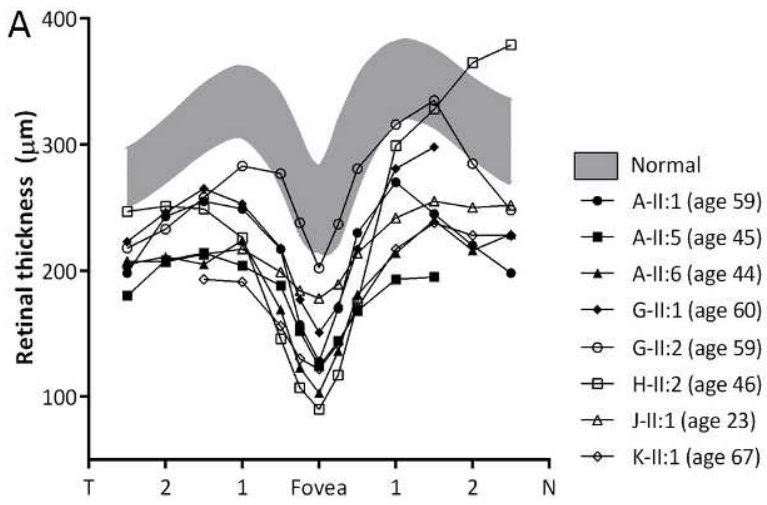

B
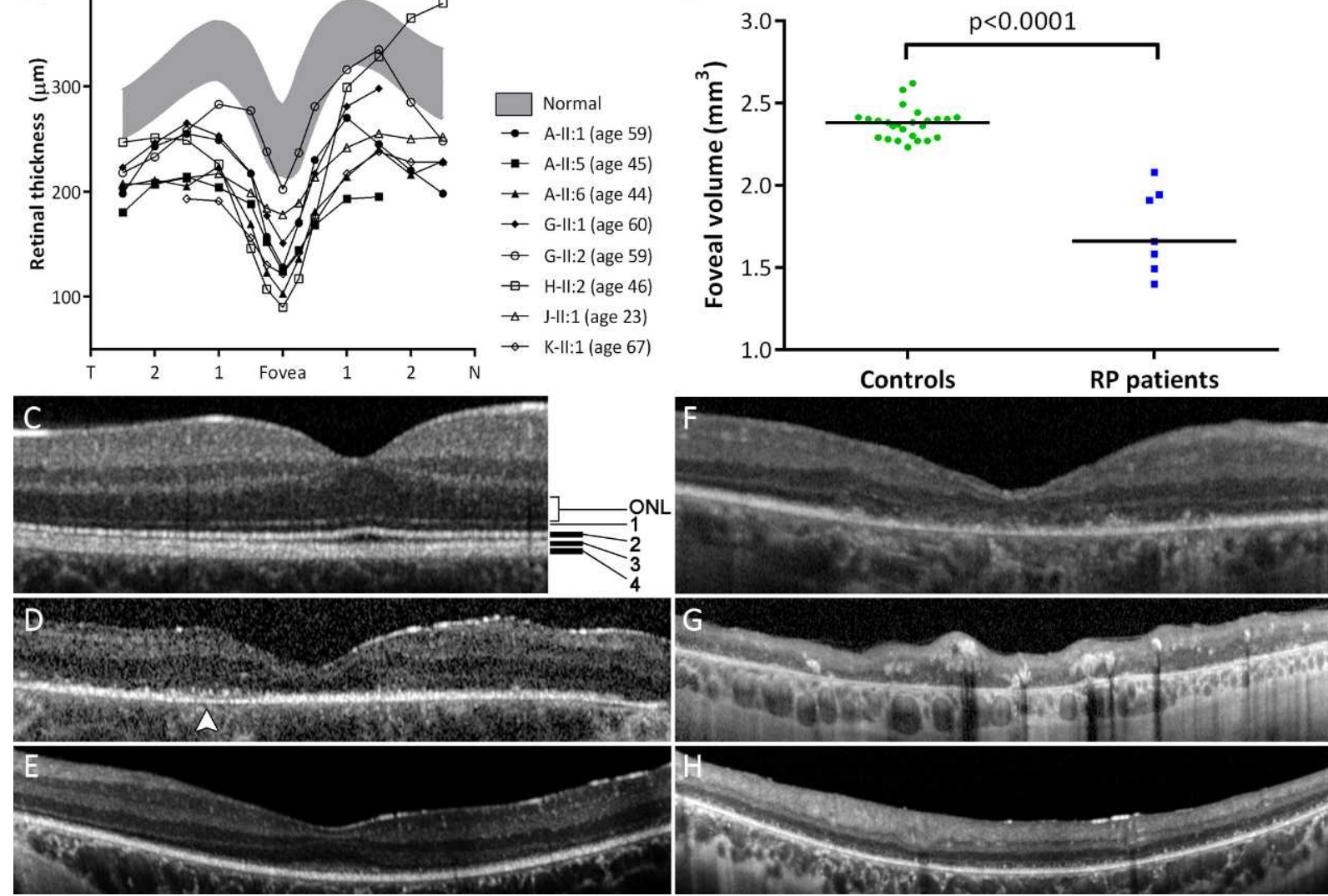

Figure 6. Optical coherence tomography examinations in patients with IMPG2-associated RP. (A) Thickness of the total retina in eight Dutch patients (mean age: 49 years; range, 23-67). Shaded areas: normal limits (mean \pm 2 SDs) as measured in 25 controls (mean age: 46 years). (B) Foveal volume $\left(\mathrm{mm}^{3}\right)$ measured in the central $3 \mathrm{~mm}^{2}$ in these patients except patient A-II:1. The foveal volume in seven Dutch IMPG2-associated RP patients (mean age: 49 years; range, 23-67) was significantly lower than in 25 age-matched healthy Dutch controls $(P<0.0001)$. The horizontal bars indicate the median of the corresponding cohort. (C) Spectral-domain OCT scan of a normal central retina (age 25). The hyperreflective bands correspond to the external limiting membrane (1), the ellipsoid photoreceptor inner segments (2), the photoreceptor outer segment/RPE contact cylinder region (3), and the RPE (4). ${ }^{24}$ (D) Spectral-domain OCT of patient A-II:6 (age 44) that reveals generalized loss of the outer retinal layers, whereas the RPE reflectance is fairly intact expect for the irregular signal and thinning in the temporal parafovea (white arrowhead). (E/H) Spectral-domain OCT in patient J-II:1 (age 23). The central retina (E) revealed normal bands corresponding to the RPE, whereas the photoreceptor bands are absent outside the fovea. The midperipheral retina $(\mathbf{H})$ reveals loss of the photoreceptor inner and outer segment bands. However, no profound RPE atrophy or intraretinal pigment deposits were observed yet. (F and G) Spectral-domain OCT of patient G-II:1 (age 60). The central retina $(\mathbf{F})$ reveals generalized loss of photoreceptor inner and outer segments, a thinned outer nuclear layer, and patchy loss/thinning of the foveal RPE. The midperipheral retina (G) shows profound loss of the outer retinal layers, RPE and choriocapillaris reflectance, as well as intraretinal pigment deposits.

eventually progresses to macular atrophy with profound degeneration of photoreceptors and RPE, although this was observed only in patient G-II:1 because longitudinal data of the other patients with macular atrophy were not available. The 13 patients with macular involvement displayed a decreased central sensitivity and/or paracentral scotomas on visual field examination in addition to the concentric constriction that is typically seen in RP (Table 1; Fig. 5). Spectral-domain OCT of the macula showed early loss of photoreceptor inner and outer segments before loss of the RPE layer in the central retina. On FAF imaging, macular hypoautofluorescence was observed, whereas hypoautofluorescence in the midperiphery generally had a granular aspect. In contrast to the significant macular involvement that was observed in most patients, subtle macular FAF abnormalities appeared in patients C-II: 2 and JII:1. However, subtle macular FAF abnormalities have been observed in other forms of RP with intact central vision and therefore do not automatically predate loss of macular function. ${ }^{25}$

A BEM is a nonspecific reaction of the posterior pole, which can occur in various diseases affecting the bipolar cell layer, photoreceptor cell layer, or RPE. ${ }^{26}$ It is not often observed in $\mathrm{RP}$, but has been reported in some specific forms of syndromic and nonsyndromic $\mathrm{RP}^{27-31}$ and is associated with a faster deterioration of the visual acuity compared with RP without specific macular lesions. ${ }^{32}$ Concerning the BEM in IMPG2associated RP, multimodal imaging revealed abnormalities in the photoreceptor and RPE cell layers. However, it is unclear why RPE abnormalities initially predominate in the perifoveal region, as the preceding abnormalities in the photoreceptor layer are ubiquitously present. Possible explanations may include topographical differences in metabolism and cell densities, ${ }^{33}$ the higher vulnerability of $S$ ("blue") cone photoreceptors to retinal disease compared with $\mathrm{M}$ and $\mathrm{L}$ cones, ${ }^{34}$ and the higher vulnerability of parafoveal rods to aging and light-induced damage. ${ }^{35-37}$ In patient G-II:2 (age 59), we observed a prominent BEM due to hypopigmentation rather than atrophy of the perifoveal RPE, as there were only minor RPE changes on FAF imaging (Fig. 4B) and mild changes of the band corresponding to the photoreceptor outer segment-RPE complex (Fig. 4C). By contrast, other patients with BEM revealed perifoveal hypoautofluorescence indicating 


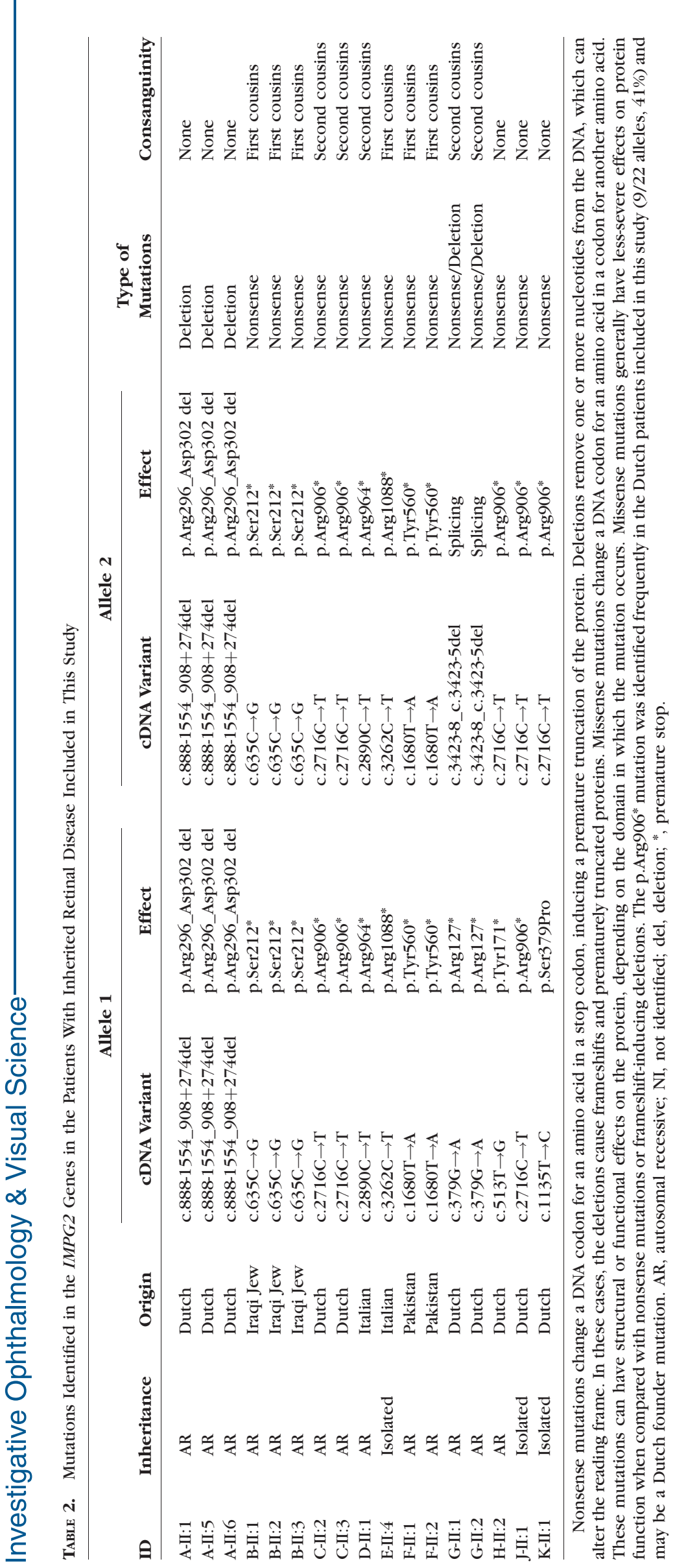


perifoveal RPE atrophy (Figs. 3E, 3G). In late stages of the disease, profound macular hypoautofluorescence developed, which is indicative of RPE atrophy (Fig. $3 \mathrm{H}$ ). In the light of future therapeutic options for retinal dystrophies, knowledge of the natural course of IMPG2-associated retinal disease is necessary to select patients amenable for treatment and to correctly interpret the effect of therapeutic intervention.

\section{Genotype-Phenotype Correlation}

Bandah-Rozenfeld et al. ${ }^{13}$ identified mutations only with severe effects on the IMPG2 protein in RP patients, whereas a homozygous mild missense mutation was identified in one patient with a mild maculopathy. In families $G$ to $K$, we identified two novel truncating nonsense mutations (families $G$ and $\mathrm{H}$ ), a deletion causing a splice defect (family $\mathrm{G}$ ), and a missense mutation that is unanimously predicted to be pathogenic (family $\mathrm{K}$ ). The function of IMPG2 is vital for retinal survival and function, as most IMPG2 mutations that are identified in our patients can be considered true loss-offunction alleles. Until now, only one homozygous mild (missense) mutation in IMPG 2 has been described in a single patient with an isolated maculopathy and a mildly affected visual function. ${ }^{13}$ This might indicate that a minimal loss of function of the IMPG2 protein may result in mild or even absent retinal disease.

Each of the seven nonsense mutations lead to either mRNA breakdown because of nonsense-mediated decay, or predicted truncated IMPG2 proteins that all lack the transmembrane domain and the cytoplasmic tail. The in-frame deletion identified in family A (c.888-1554_908+274del; absence of seven amino acids) is thought to result in a nonfunctional IMPG2 protein, as in a cellular transfection assay this mutant protein was retained in the endoplasmic reticulum, whereas IMPG2 is physiologically located in the cell membrane. ${ }^{13}$ The 4-base-pair deletion of the splice acceptor site in family $G$ (c.3423-8_c.3423-5del) was found to result in the use of an alternative splice acceptor site, and thereby also predicted to result in the generation of a truncated protein that most likely has reduced or no remaining function. Interestingly, the p.Arg906* was present in 8 of 20 alleles (40\%) in 10 Dutch patients, which may imply a founder mutation in the Dutch population.

Because the identified mutations cause (near-)complete loss of IMPG2 function, the clinical variation is limited in IMPG2associated RP. The patients in family G, however, retained slightly better visual acuity and visual field compared with the other patients (Fig. 2; Table 1). This could imply that the splice defect in this family results in an IMPG2 protein with some residual function. However, functional assays are needed to reveal the true effect of the c.3423-8_c.3423-5 deletion, as there also is evidence of modifying factors in this family (Table 1; Figs. 2, 3B, 3H, 4) that influence the intrafamilial differences.

The IMPG2 protein (SPACRCAN) is highly homologous to SPACR, the product of the interphotoreceptor matrix proteoglycan 1 (IMPG1) gene, which has been linked to benign concentric annular macular dystrophy (BCAMD) and vitelliform macular dystrophy (VMD). ${ }^{38,39}$ Interestingly, BCAMD includes parafoveal hypopigmentation and RP-like fundoscopic changes in the end-stage disease, although visual acuity is generally better preserved than in the IMPG2-associated RP patients in this report. ${ }^{38}$ The IMPG1-associated vitelliform dystrophy is also associated with macular pathology, although this is characterized by accumulation of lipofuscin rather than a BEM. ${ }^{39}$

In conclusion, severe mutations in IMPG2 are the cause of an autosomal recessive RP phenotype that manifests in the early teens and is accompanied by atrophic maculopathy often in a bull's eye pattern. In early disease stages, the maculopathy is characterized by mild RPE alterations, but in later stages of the disease, a BEM and profound macular chorioretinal atrophy may occur. In most patients, the RP phenotype arising from mutations in the IMPG2 gene is severe, because of the unfortunate combination of progressive constriction of the visual fields and maculopathy that occurs relatively early in the course of the disease.

\section{Acknowledgments}

We thank all patients included in this study. We also thank Linda Visser and Annemiek Krijnen for their help regarding the clinical data of the Dutch patients from the Rotterdam Eye Hospital.

Supported by the Stichting A.F. Deutman Researchfonds Oogheelkunde, Nijmegen, The Netherlands (BJK), Grants BR-GE-05100489-RAD (AIdH) and C-GE-0811-0545-RAD01 from the Foundation Fighting Blindness USA (FPMC, RWJC, AIdH), the Stichting Wetenschappelijk Onderzoek Het Oogziekenhuis Prof Dr H.J. Flieringa (LIvdB, AIdH, FPMC), and The Netherlands Organization for Health Research and Development (ZonMW; TOP-Grant 4000812-98-09047 [AIdH, FPMC]). The funding organizations had no role in the design or conduct of this research.

Disclosure: R.A.C. van Huet, None; R.W.J. Collin, None; A.M. Siemiatkowska, None; C.C.W. Klaver, None; C.B. Hoyng, None; F. Simonelli, None; M.I. Khan, None; R. Qamar, None; E. Banin, None; F.P.M. Cremers, None; T. Theelen, None; A.I. den Hollander, None; L.I. van den Born, None; B.J. Klevering, None

\section{References}

1. Bunker CH, Berson EL, Bromley WC, Hayes RP, Roderick TH. Prevalence of retinitis pigmentosa in Maine. Am J Ophthalmol. 1984;97:357-365.

2. Rosenberg T. Epidemiology of hereditary ocular disorders. Dev Ophthalmol. 2003;37:16-33.

3. Berson EL. Retinitis pigmentosa. The Friedenwald Lecture. Invest Ophthalmol Vis Sci. 1993;34:1659-1676.

4. Hartong DT, Berson EL, Dryja TP. Retinitis pigmentosa. Lancet. 2006;368:1795-1809.

5. Hamel C. Retinitis pigmentosa. Orphanet J Rare Dis. 2006;1: 40.

6. Dryja TP, Hahn LB, Kajiwara K, Berson EL. Dominant and digenic mutations in the peripherin/RDS and ROM1 genes in retinitis pigmentosa. Invest Ophthalmol Vis Sci. 1997;38: 1972-1982.

7. den Hollander AI, Roepman R, Koenekoop RK, Cremers FP. Leber congenital amaurosis: genes, proteins and disease mechanisms. Prog Retin Eye Res. 2008;27:391-419.

8. Schrier SA, Falk MJ. Mitochondrial disorders and the eye. Curr Opin Ophthalmol. 2011;22:325-331.

9. Kajiwara K, Berson EL, Dryja TP. Digenic retinitis pigmentosa due to mutations at the unlinked peripherin/RDS and ROM1 loci. Science. 1994;264:1604-1608.

10. den Hollander AI, Black A, Bennett J, Cremers FP. Lighting a candle in the dark: advances in genetics and gene therapy of recessive retinal dystrophies. J Clin Invest. 2010;120:30423053.

11. Neveling K, Feenstra I, Gilissen C, et al. A post-hoc comparison of the utility of sanger sequencing and exome sequencing for the diagnosis of heterogeneous diseases. Hum Mutat. 2013;34:1721-1726.

12. Berger W, Kloeckener-Gruissem B, Neidhardt J. The molecular basis of human retinal and vitreoretinal diseases. Prog Retin Eye Res. 2010;29:335-375. 
13. Bandah-Rozenfeld D, Collin RW, Banin E, et al. Mutations in IMPG2, encoding interphotoreceptor matrix proteoglycan 2 , cause autosomal-recessive retinitis pigmentosa. Am J Hum Genet. 2010;87:199-208.

14. Inatani M, Tanihara H. Proteoglycans in retina. Prog Retin Eye Res. 2002;21:429-447.

15. Adler AJ, Klucznik KM. Proteins and glycoproteins of the bovine interphotoreceptor matrix: composition and fractionation. Exp Eye Res. 1982;34:423-434.

16. Hageman GS, Johnson LV. Structure, composition and function of the retinal interphotoreceptor matrix. In: Osborne $\mathrm{N}$, Chader G, eds. Retinal Research. New York: Pergamon Press; 1991:207-249.

17. Berman ER. Mucopolysaccharides (glycosaminoglycans) of the retina: identification, distribution and possible biological role. Bibl Ophthalmol. 1969;79:5-31.

18. Hewit AT, Adler R. The retinal pigment epithelium and interphotoreceptor matrix: structure and specialized function. In: Ryan SJ, ed. Retina. St. Louis: CV Mosby Co.; 1989:57-64.

19. Enoch JM, Laties AM. An analysis of retinal receptor orientation. II. Predictions for psychophysical tests. Invest Ophthalmol. 1971;10:959-970.

20. Acharya S, Foletta VC, Lee JW, et al. SPACRCAN, a novel human interphotoreceptor matrix hyaluronan-binding proteoglycan synthesized by photoreceptors and pinealocytes. J Biol Chem. 2000;275:6945-6955.

21. Chen Q, Cai S, Shadrach KG, Prestwich GD, Hollyfield JG. Spacrcan binding to hyaluronan and other glycosaminoglycans. Molecular and biochemical studies. J Biol Chem. 2004; 279:23142-23150.

22. Neveling $\mathrm{K}$, Collin RW, Gilissen $\mathrm{C}$, et al. Next generation genetic testing for retinitis pigmentosa. Hum Mutat. 2012;33: 963-972.

23. Marmor MF, Fulton AB, Holder GE, Miyake Y, Brigell M, Bach MISCEV. Standard for full-field clinical electroretinography (2008 update). Doc Ophthalmol. 2009;118:69-77.

24. Spaide RF, Curcio CA. Anatomical correlates to the bands seen in the outer retina by optical coherence tomography: literature review and model. Retina. 2011;31:1609-1619.

25. Makiyama $Y$, Ooto S, Hangai $M$, et al. Macular cone abnormalities in retinitis pigmentosa with preserved central vision using adaptive optics scanning laser ophthalmoscopy. PLoS One. 2013;8:e79447.

26. Pinckers A, Cruysberg JR, van de Kerk AL. Main types of bull's eye maculopathy. Functional classification. Doc Ophthalmol. 1984;58:257-267.
27. Campo RV, Aaberg TM. Ocular and systemic manifestations of the Bardet-Biedl syndrome. Am J Ophthalmol. 1982;94:750756.

28. Koenekoop RK, Loyer M, Hand CK, et al. Novel RPGR mutations with distinct retinitis pigmentosa phenotypes in French-Canadian families. Am J Opbthalmol. 2003;136:678687.

29. Kikawa E, Nakazawa M, Chida Y, Shiono T, Tamai M. A novel mutation (Asn244Lys) in the peripherin/RDS gene causing autosomal dominant retinitis pigmentosa associated with bull's-eye maculopathy detected by nonradioisotopic SSCP. Genomics. 1994;20:137-139.

30. Demirci FY, Gupta N, Radak AL, et al. Histopathologic study of $\mathrm{X}$-linked cone-rod dystrophy (CORDX1) caused by a mutation in the RPGR exon ORF15. Am J Ophthalmol. 2005;139:386388.

31. Michaelides M, Gaillard MC, Escher P, et al. The PROM1 mutation p.R373C causes an autosomal dominant bull's eye maculopathy associated with rod, rod-cone, and macular dystrophy. Invest Ophthalmol Vis Sci. 2010;51:4771-4780.

32. Flynn MF, Fishman GA, Anderson RJ, Roberts DK. Retrospective longitudinal study of visual acuity change in patients with retinitis pigmentosa. Retina. 2001;21:639-646.

33. Curcio CA, Sloan KR, Kalina RE, Hendrickson AE. Human photoreceptor topography. J Comp Neurol. 1990;292:497523.

34. Greenstein VC, Hood DC, Ritch R, Steinberger D, Carr RE. S (blue) cone pathway vulnerability in retinitis pigmentosa, diabetes and glaucoma. Invest Ophthalmol Vis Sci. 1989;30: 1732-1737.

35. Curcio CA. Photoreceptor topography in ageing and agerelated maculopathy. Eye (Lond). 2001;15:376-383.

36. Curcio CA, Millican CL, Allen KA, Kalina RE. Aging of the human photoreceptor mosaic: evidence for selective vulnerability of rods in central retina. Invest Ophthalmol Vis Sci. 1993;34:3278-3296.

37. Okano K, Maeda A, Chen Y, et al. Retinal cone and rod photoreceptor cells exhibit differential susceptibility to lightinduced damage. J Neurochem. 2012;121:146-156.

38. van Lith-Verhoeven JJ, Hoyng CB, van den Helm B, et al. The benign concentric annular macular dystrophy locus maps to 6p12.3-q16. Invest Ophthalmol Vis Sci. 2004;45:30-35.

39. Manes G, Meunier I, Avila-Fernandez A, et al. Mutations in IMPG1 cause vitelliform macular dystrophies. Am J Hum Genet. 2013;93:571-578. 